\title{
Intestinal healing after anti-TNF induction therapy predicts long-term response to one-year treatment in patients with ileocolonic Crohn's disease naive to anti-TNF agents
}

Piotr Eder ${ }^{1}$, Liliana Łykowska-Szuber ${ }^{1}$, Katarzyna Katulska², Kamila Stawczyk-Eder ${ }^{1}$, Iwona Krela-Kaźmierczak ${ }^{1}$, Katarzyna Klimczak ${ }^{1}$, Aleksandra Szymczak ${ }^{1}$, Marek Stajgis ${ }^{2}$, Krzysztof Linke ${ }^{1}$

${ }^{1}$ Department of Gastroenterology, Human Nutrition and Internal Diseases, Poznan University of Medical Sciences, Poznan, Poland

2Department of Radiology, Poznan University of Medical Sciences, Poznan, Poland

Key words: anti-TNF antibodies, Crohn's disease, colonoscopy, magnetic resonance enterography.

Address for correspondence: Piotr Eder MD, PhD, Department of Gastroenterology, Human Nutrition and Internal Diseases, Poznan University of Medical Sciences, 49 Przybyszewskiego St, 60-355 Poznan, Poland, phone: +48 698 050 797, e-mail: piotr.eder@op.pl

\begin{abstract}
Introduction: Objective assessment of Crohn's disease (CD) activity in patients treated with anti-tumour necrosis factor (anti-TNF) antibodies is crucial for the prediction of its long-term results. Mucosal healing estimated endoscopically has a strong predictive value; however, only combined assessment together with transmural healing in magnetic resonance enterography (MRE) gives full information about the whole spectrum of inflammatory lesions in CD.

Aim: To assess the usefulness of intestinal healing phenomenon in CD, defined as improvement both in endoscopy and MRE, after anti-TNF induction therapy, in predicting long-term results of 1-year treatment.

Material and methods: Twenty-six patients with ileocolonic CD were enrolled into the study. In this group a parallel assessment of disease activity was estimated before and after induction doses of anti-TNF antibodies with ileocolonoscopy and MRE by using appropriate scores. Subsequently the patients were treated until 12 months and then followed-up. The associations between intestinal healing (assessed in MRE and endoscopy), and mucosal and transmural healing with long-term results of 1 -year anti-TNF therapy were analysed statistically.

Results: The median time of follow-up was 29 months (interquartile range - IQR: 14-46). Intestinal healing was significantly associated with favourable therapeutic outcomes $(p=0.02)$ and had 75\% (IQR: $35-97 \%)$ sensitivity and 72\% (IQR: 46-90\%) specificity in predicting long-term remission. Other parameters were not useful (transmural healing) or their usefulness was of borderline significance (mucosal healing).

Conclusions: Dynamic assessment of intestinal healing is an accurate method in predicting long-term outcomes in CD patients responding to 1-year anti-TNF therapy.
\end{abstract}

\section{Introduction}

Predicting the long-term efficacy of anti-tumour necrosis factor $\alpha$ (anti-TNF) therapy in Crohn's disease $(C D)$ is essential for the optimisation of this expensive immunomodulating treatment. Several factors have been investigated in this field; however, there are still a lot of unanswered questions [1, 2].

Mucosal healing is believed to be a very important phenomenon, which predicts, with probably the highest accuracy, the successful long-term application of biological therapy [3]. However, it is still debated how this phenomenon should be defined [3, 4]. Moreover, endoscopic assessment allows only for the visualisation of the mucosal surface of the intestine, whereas inflammatory lesions in CD concern the whole gut wall and very often also the mesenteric fatty tissue. Thus, new cross-sectional imaging methods, which enable the detection of transmural CD activity, are an essential diagnostic complementation for gastrointestinal endoscopy. The most commonly used radiologic 
technique is magnetic resonance enterography (MRE) [5]. Magnetic resonance enterography is a very useful tool in the assessment of the transmural healing in CD patients treated with immunosuppressive drugs, because, as it is a radiation-free method, it can be performed multiple times in the same patient $[5,6]$. Nevertheless, MRE also has its drawbacks. One of them is that it does not allow the detection of subtle and very early mucosal erosions and ulcerations, which can only be detected during endoscopy [6]. Thus, it seems that both diagnostic methods, endoscopy and MRE, are not competitive but complementary tools in the assessment of ileal and ileocolonic CD activity, and preferably they should be performed parallel. As mucosal healing in patients treated with anti-TNF antibodies seems to be predictive of successful application of biological maintenance therapy, it can be hypothesised, that a more complex and objective assessment could be even more accurate. However, such analysis has not been performed yet.

\section{Aim}

The main aim of the current study was to answer the question of whether a combined assessment of CD activity using both ileocolonoscopy and MRE (intestinal healing phenomenon) after induction doses of anti-TNF agents can be more accurate in predicting long-term response to 1-year biological therapy than endoscopic (mucosal healing) or radiologic (transmural healing) assessment alone.

\section{Material and methods}

Patients with diagnosed CD, who were qualified for the first time for anti-TNF therapy between 09.2009 and 09.2013 at our Department were enrolled into the study. All data were prospectively collected and analysed retrospectively. The inclusion criteria for analysis were as follows: age $\geq 18$ years, ileocolonic CD location, exacerbation of CD not responding to maximal doses of a standard therapy (mesalamine, thiopurines, steroids, and/or antibiotics) or intolerance/side effects of a standard therapy, full ileocolonoscopy and MRE performed twice: 0-3 weeks before enrolment and, for the second time, after induction doses of anti-TNF agents, primary response to the induction doses of anti-TNF agents and successful continuation of biological therapy until week 52-54 (1-year anti-TNF therapy).

The exclusion criteria were: previous anti-TNF therapy, significant change in the therapeutic regimen (introducing new drug, increasing the dosage of concomitant therapy) during anti-TNF induction treatment, primary (during the induction doses) or secondary (during maintenance therapy) loss of response to anti-TNF agents, loss to long-term follow-up after finishing 1-year of biological therapy.

All patients were treated according to the current Polish guidelines for biological therapy in CD [7]. Infliximab (IFX) was administered intravenously at $5 \mathrm{mg} / \mathrm{kg}$ body weight at week 0,2 , and 6 and then every 8 weeks until week 52-54. Adalimumab (ADA) was administered subcutaneously at week $0-160 \mathrm{mg}$, at week 2-80 mg, and then every other week - $40 \mathrm{mg}$ until week 52-54.

The clinical, biochemical, endoscopic, and radiologic activity of CD were assessed before starting anti-TNF therapy and after induction doses of biological agents - at week 12-14 in case of ADA, and at week 9-12 in case of IFX.

Clinical activity was estimated by using Crohn's Disease Activity Index (CDAI) [8]. The main outcomes assessed in the study were: mucosal, transmural, and intestinal healing in the course of anti-TNF therapy, estimated during ileocolonoscopy and MRE investigations. lleocolonoscopy was performed by an experienced endoscopist with the estimation of CD activity done by calculating the Simple Endoscopic Score for CD (SES-CD) [9]. Mucosal healing was defined as a $\geq 50 \%$ decrease in SES-CD score. MRE was performed according to a standard protocol, described previously [10]. All MRE data were analysed and quantified by an experienced radiologist with more than 13 years of experience in this cross-sectional imaging method. The activity of CD in MRE was assessed by using the Simple Enterographic Activity Score for CD (SEAS-CD) [10]. Transmural healing was defined as a $\geq 50 \%$ decrease in SEAS-CD score.

To define intestinal healing we combined the endoscopic and radiologic assessment. This phenomenon was defined as a $\geq 50 \%$ decrease in both SES-CD and SEAS-CD after induction doses of anti-TNF agents.

After finishing 1-year anti-TNF therapy, all patients were followed-up until 02.2015. Exacerbation of disease was defined as a recurrence of CD symptoms needing introduction of steroids in ambulatory care, or needing hospitalisation. Long-term response was defined as a remission (CDAl < 150 pts) up to the end of the follow-up period.

The simplified protocol of the study is presented in Figure 1.

\section{Statistical analysis}

Data are presented as medians with interquartile ranges (IQR). All data were analysed using non-parametric (Wilcoxon test) or parametric (paired $t$ test) statistics for paired data, as appropriate. Categorised data were assessed with the $\chi^{2}$ test. Correlations were assessed with the use of Spearman's rank correlation coefficient. Sensitivity, specificity, as well as negative 


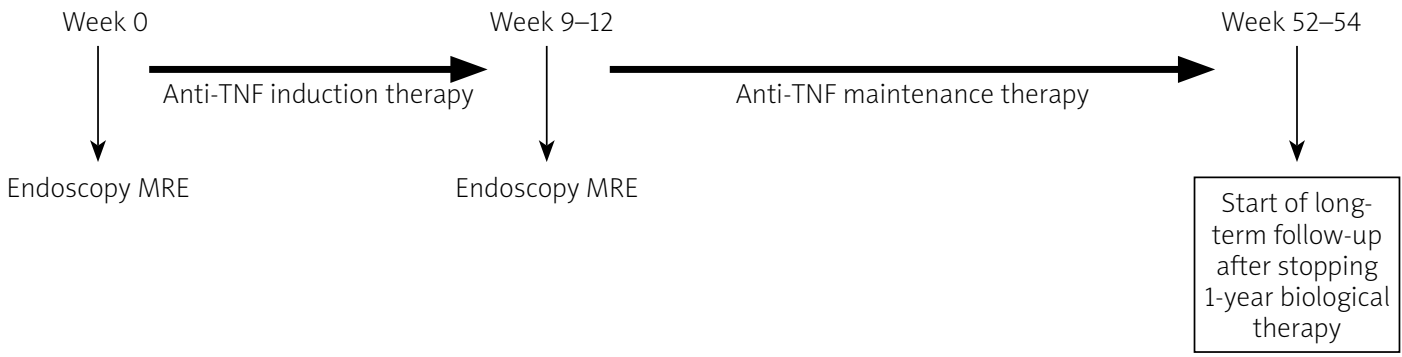

Figure 1. The simplified protocol of the study. The activity of Crohn's disease (CD) was assessed twice before and after anti-TNF induction therapy. The activity was assessed with colonoscopy and magnetic resonance enterography using appropriate scores. All primary responders were treated with maintenance doses of biological agents till week 52-54. Then all patients were followed-up and percentages of patients who exacerbated or who achieved long-term remission during this period were analysed

and positive predictive values (NPV and PPV, respectively) were also calculated for predicting long-term results of anti-TNF therapy in CD patients who achieved mucosal, transmural, and intestinal healing after induction doses of biological agents. A $p$ value $<0.05$ was considered significant. All data were analysed using GraphPad Prism Version 6.0 (GraphPad Software Inc., USA).

\section{Ethical considerations}

The study was approved by the Bioethics Committee of Poznan University of Medical Sciences (decision no. 774/2013).

\section{Results}

Among 103 patients treated with anti-TNF agents between 09.2009 and 09.2013 in our Department, 26 adhered to the inclusion and exclusion criteria (10 males, 16 females, median age -27 years (IQR: $21-36)$ ). The median follow-up period after finishing 1-year anti-TNF therapy was 29 months (IQR: 14-46). Ten $(38 \%)$ patients sustained remission in long-term follow-up period, whereas 16 (62\%) individuals experienced CD exacerbation. The baseline characteristics of the study group are presented in Table I.

Table II shows how the most important parameters assessing CD activity changed after anti-TNF induction therapy. Only white blood cell count did not change significantly. Both the endoscopic (SES-CD) as well the enterographic (SEAS-CD) activity decreased, achieving statistical significance.

After finishing the anti-TNF induction therapy, we observed transmural healing, mucosal healing, and intestinal healing in the case of 10 (38\%), 16 (62\%), and $8(31 \%)$ patients, respectively.

Numbers of patients achieving and not achieving transmural healing after induction biological therapy, who sustained remission or exacerbated in long-term observation, are shown in Figure 2. Transmural healing phenomenon was favourable for long-term remission; however, this trend was not statistically significant $(p=0.08)$ (Figure 1). Transmural healing had 60\% (IQR: 26-88\%) sensitivity and 75\% (IQR: 47-92\%) specificity in predicting long-term remission. PPV was 60\% (IQR: 26-88\%) and NPV was 75\% (IQR: 47-92\%).

Numbers of patients achieving and not achieving mucosal healing after induction biological therapy, who sustained remission or exacerbated in long-term observation are shown in Figure 3. Mucosal healing phenom-

Table I. Baseline characteristics of the study group 26 Crohn's disease patients with ileocolonic location of the disease. Data are presented as medians with interquartile ranges (IQR)

\begin{tabular}{|c|c|}
\hline Feature & Results \\
\hline Age [years] & 27 (IQR: 21-36) \\
\hline Months of follow-up & 29 (IQR: 14-46) \\
\hline Male/female, $n$ & $10 / 16$ \\
\hline Exacerbation during follow-up period, $n(\%)$ & $16(62)$ \\
\hline Disease duration [years] & 4 (IQR: 2-6) \\
\hline \multicolumn{2}{|l|}{ Disease behaviour, $n(\%)$ : } \\
\hline B1 (inflammatory) & $16(62)$ \\
\hline B2 (stricturing) & $2(7)$ \\
\hline B3 (penetrating) & $8(31)$ \\
\hline \multicolumn{2}{|l|}{ Medications, $n(\%)$ : } \\
\hline Steroids & $23(88)$ \\
\hline Azathioprine & $23(88)$ \\
\hline Amino salicylates & $26(100)$ \\
\hline Antibiotics & $14(54)$ \\
\hline $\begin{array}{l}\text { Anti-TNF agent used: } \\
\text { adalimumab/infliximab, } n(\%)\end{array}$ & $6(24) / 20(76)$ \\
\hline
\end{tabular}


Table II. The influence of the anti-TNF induction therapy on the most important parameters assessing the activity of Crohn's disease. Data are presented as medians with interquartile ranges (IQR)

\begin{tabular}{|c|c|c|c|}
\hline Feature & Before therapy & $\begin{array}{l}\text { After induction } \\
\text { therapy }\end{array}$ & $P$-value \\
\hline Simple Enterographic Activity Score for Crohn's Disease [points] & 12 (IQR: 10-16) & 7 (IQR: 5-11) & $<0.0001$ \\
\hline C-reactive protein [mg/l] & 9.9 (IQR: 1.9-40) & 2.3 (IQR: 1-8.2) & 0.004 \\
\hline Erythrocyte sedimentation rate $[\mathrm{mm} / \mathrm{h}]$ & 29 (IQR: 15-54) & 20 (IQR: 11-32) & 0.02 \\
\hline Haemoglobin [g/dl] & 11.9 (IQR: 9.4-12.7) & 12.1 (IQR: 11.1-13.9) & 0.009 \\
\hline White blood cell count $\left[10^{3} / \mathrm{mm}^{3}\right]$ & 6.3 (IQR: 4.9-8.3) & 5.8 (IQR: 4.2-7.1) & 0.14 \\
\hline Platelets $\left[10^{3} / \mathrm{mm}^{3}\right]$ & 363 (IQR: 246-447) & 285 (IQR: 230-375) & 0.001 \\
\hline Crohn's Disease Activity Index [points] & 302 (IQR: 224-344) & 85 (IQR: 50-194) & $<0.0001$ \\
\hline Simple Endoscopic Score for Crohn's Disease [points] & 13 (IQR: 6-19) & $4(\mathrm{IQR}: 3-5)$ & $<0.0001$ \\
\hline
\end{tabular}

enon was favourable for long-term remission, and this trend had a borderline significance in statistical analysis $(p=0.06)$ (Figure 2). Mucosal healing had 50\% (IQR: 24-75\%) sensitivity and 80\% (IQR: 44-97\%) specificity in predicting long-term remission. PPV was $80 \%$ (IQR: 44-97\%) and NPV was 50\% (IQR: 24-75\%).

Numbers of patients achieving and not achieving intestinal healing after induction biological therapy, who sustained remission or exacerbated in long-term observation are shown in Figure 4. Intestinal healing phenomenon was favourable for long-term remission, and this trend was statistically significant $(p=0.02)$ (Figure 3). Intestinal healing had 75\% (IQR: 35-97\%) sensitivity and 72\% (IQR: 46-90\%) specificity in predict-

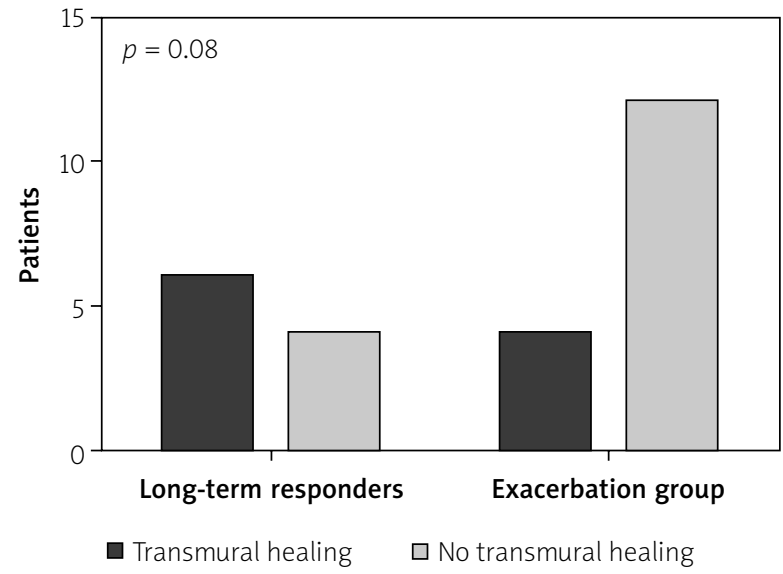

Figure 2. Numbers of patients achieving and not achieving transmural healing after induction biological therapy, who sustained remission or exacerbated in long-term observation. Transmural healing phenomenon was favourable for longterm remission; however, this trend was not statistically significant $(p=0.08)$ ing long-term remission. PPV was 54\% (IQR: 23-83\%) and NPV was $87 \%$ (IQR: 59-98\%).

We also performed comparative analysis of differences between chosen characteristics among patients who sustained long-term remission and who experienced exacerbation during the follow-up period (Table III). The median duration of follow-up in longterm responders was 45 months (IQR: 26-49). Median duration of remission in the exacerbation group after 1 -year of successful anti-TNF therapy was 18 months (IQR: 10-34). Only baseline haemoglobin levels were significantly lower in the exacerbation group when compared with long-responders. We observed, however, a trend of higher disease activity (assessed clinically,

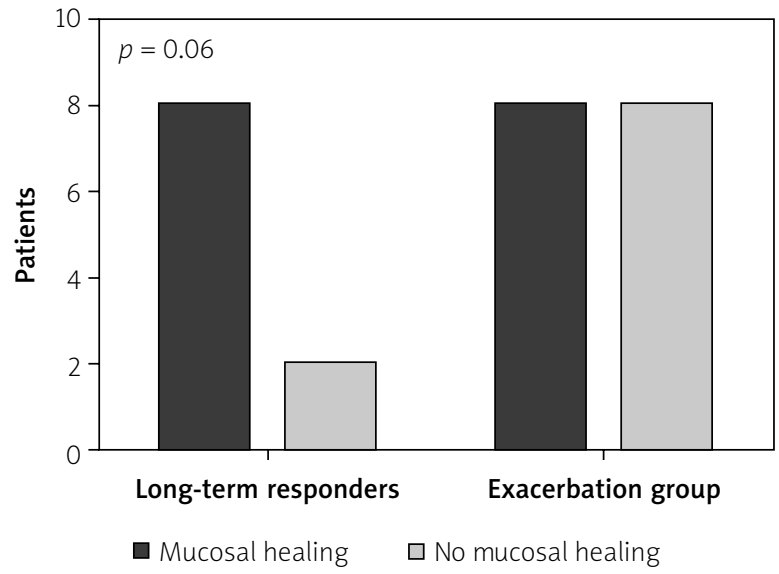

Figure 3. Numbers of patients achieving and not achieving mucosal healing after induction biological therapy, who sustained remission or exacerbated in long-term observation. Mucosal healing phenomenon was favourable for longterm remission, and this trend had a borderline significance in statistical analysis $(p=0.06)$ 
biochemically, endoscopically, as well as in MRE) both at baseline and after anti-TNF induction therapy in the exacerbation group, although these differences were not statistically significant.

\section{Discussion}

Dynamic assessment of disease course in CD patients treated with anti-TNF agents is crucial for appropriate monitoring of treatment outcomes [4]. The majority of data in this respect concern the endoscopic evaluation of mucosal healing; however, the number of studies in this field is still limited. Nevertheless, it is believed that endoscopy not only reliably informs clinicians about the current activity of the disease in a single patient, but also it can be helpful in predicting long-term outcomes of the therapy [2-4]. Baert et al. were the first to show that mucosal healing predicted sustained clinical remission in patients with early-stage CD [11]. In this prospective trial complete mucosal healing was the only factor connected with steroid-free remission 3 and 4 years after the therapy was initiated.

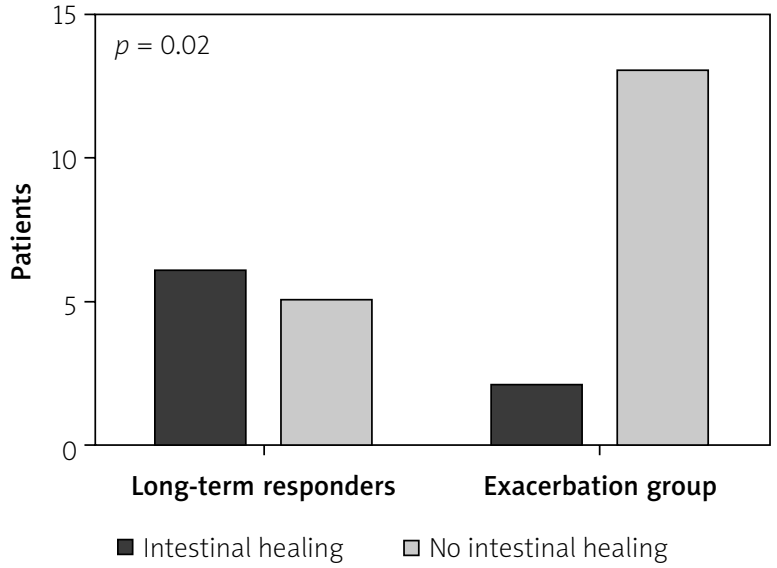

Figure 4. Numbers of patients achieving and not achieving intestinal healing after induction biological therapy, who sustained remission or exacerbated in long-term observation. Intestinal healing phenomenon was favourable for longterm remission, and this trend was statistically significant $(p=0.02)$

Table III. Comparison of chosen data between long-term responders and the exacerbation group. Data are presented as medians with interquartile ranges (IQR)

\begin{tabular}{|c|c|c|c|}
\hline Feature & $\begin{array}{l}\text { Long-term } \\
\text { responders }(n=10)\end{array}$ & $\begin{array}{l}\text { Exacerbation group } \\
\qquad(n=16)\end{array}$ & $P$-value \\
\hline Age [years] & 27 (IQR: 24-33) & 27 (IQR: 19-36) & 0.83 \\
\hline Male/female, $n$ & $4 / 6$ & $6 / 10$ & 0.89 \\
\hline Disease duration [years] & 5 (IQR: 3-8) & $4(I Q R: 2-6)$ & 0.76 \\
\hline Months of follow-up & 45 (IQR: 26-49) & 18 (IQR: 10-34) & 0.04 \\
\hline Baseline Crohn's Disease Activity Index [points] & 267 (IQR: 203-374) & 303 (IQR: 261-332) & 0.87 \\
\hline Baseline Simple Endoscopic Score for Crohn's Disease [points] & $12(\mathrm{IQR}: 7-23)$ & 13 (IQR: 5-18) & 0.39 \\
\hline Baseline C-reactive protein [mg/l] & 2.9 (IQR: 1.7-15.6) & 17.8 (IQR: 4-50) & 0.3 \\
\hline Baseline haemoglobin [g/dl] & 12.2 (IQR: 11.3-12.9) & 11.2 (IQR: 9.2-12.4) & 0.02 \\
\hline Baseline platelets $\left[10^{3} / \mathrm{mm}^{3}\right]$ & 287 (IQR: 235-448) & 373 (IQR: 294-446) & 0.51 \\
\hline Baseline erythrocyte sedimentation rate $[\mathrm{mm} / \mathrm{h}]$ & 15 (IQR: 8-34) & 49 (IQR: 25-59) & 0.2 \\
\hline Baseline Simple Enterographic Activity Score for Crohn's Disease [points] & $11($ IQR: 9-12) & 13 (IQR: 12-16) & 0.12 \\
\hline Crohn's Disease Activity Index after induction therapy [points] & 50 (IQR: 45-113) & 138 (IQR: 67-218) & 0.06 \\
\hline Simple Endoscopic Score for Crohn's Disease after induction therapy [points] & $4(\mathrm{IQR}: 3-6)$ & 5 (IQR: $3-5)$ & 0.75 \\
\hline C-reactive protein after induction therapy $[\mathrm{mg} / \mathrm{l}]$ & $1.6(\mathrm{IQR}: 1.1-13.1)$ & 2.7 (IQR: 0.9-6.5) & 0.99 \\
\hline Haemoglobin after induction therapy $[\mathrm{g} / \mathrm{dl}]$ & 12.4 (IQR: 11.7-14.1) & 11.6 (IQR: 10.6-13.8) & 0.41 \\
\hline Platelets after induction therapy $\left[10^{3} / \mathrm{mm}^{3}\right]$ & 299 (IQR: 228-388) & 282 (IQR: 225-368) & 0.42 \\
\hline Erythrocyte sedimentation rate after induction therapy $[\mathrm{mm} / \mathrm{h}]$ & 13 (IQR: 7-30) & 22 (IQR: 15-45) & 0.28 \\
\hline SEAS-CD after induction therapy & 6 (IQR: 3-8) & 9 (IQR: 5-12) & 0.12 \\
\hline
\end{tabular}


Moreover, they showed that the majority of patients (15 of $17 ; 88 \%$ ) with complete mucosal healing at year 2 maintained remission without any further IFX infusions during years 3 and 4 [11]. Another study, performed by Schnitzler et al., provided further data on the predictive role of mucosal healing for improved longterm outcome of CD treated with IFX [12]. They showed that endoscopic improvement was especially associated with lower need for major abdominal surgery during long-term follow-up. Finally, in the most recent study from Bjorkesten et al., in which 33 CD patients treated for 1-year with anti-TNF agents were included, it was suggested that mucosal healing assessed at 3 months after starting the therapy had sensitivity of $88 \%$ and specificity of $64 \%$ to predict long-term endoscopic remission [13].

The MRE is another diagnostic tool with confirmed usefulness in the assessment of CD activity [14]. It has also been suggested that it could be helpful in monitoring the efficacy of anti-TNF therapy, as it allows assessment of the influence of biological agents on the whole gut wall and its surroundings (transmural healing) [15]. However, the prognostic value of transmural healing seen in MRE for long-term outcomes of anti-TNF treatment has not been investigated so far.

As was mentioned above, although endoscopy seems to be the most sensitive tool in the assessment of $C D$ activity, it does not allow the evaluation of the whole spectrum of transmural inflammatory lesions. Such assessment is possible in MRE, but this cross-sectional imaging method is less accurate in detecting subtle mucosal ulcerations and/or erosions. Thus, combining of these two methods (endoscopy and MRE) is reasonable, as it provides complete and full data on the activity of CD. That is why we wanted to assess whether such a diagnostic approach can be more accurate in the prognosis of long-term outcomes of anti-TNF therapy when compared with endoscopic or radiologic (MRE) assessment alone. In order to perform such an analysis, only CD patients with ileocolonic location, in whom a parallel endoscopic and radiologic assessment of disease activity in the same intestinal segments was possible, were enrolled into the study. As the aforementioned combined analysis of disease activity provides data on the influence of biological treatment on the whole spectrum of inflammatory lesions in $C D$, we called it intestinal healing, in contrast to mucosal (assessed endoscopically) and transmural (assessed in MRE) healing.

We showed that intestinal healing after finishing the induction phase of anti-TNF therapy is significantly predictive of long-term remission after stopping 1-year biological treatment. The median duration of remission after finishing biological therapy in our study group among long-responders was 45 months. On the other hand, the lack of intestinal healing, even if the patient had clinical, endoscopic, or radiologic improvement, was associated with an almost $90 \%$ probability of another exacerbation in a short period of time after stopping 1-year anti-TNF treatment.

These data are very important for everyday clinical practice, especially in countries with restricted access to biological therapy. For example, in Poland only 1-year treatment in CD patients is possible. Knowledge about the probable further disease course after 12 months of biological therapy could help clinicians to decide how strict the diagnostic surveillance should be. One can hypothesise that, for example, patients with no intestinal healing should undergo more restrictive assessment (measurement of faecal calprotectin levels and/or MRE and/or endoscopy) to detect disease flare in its early phase. Another option would be to prolong the anti-TNF treatment over 12 months in patients without intestinal healing to prevent early disease exacerbation. In contrast to that, in the case of patients achieving intestinal healing, stopping the therapy after 1 -year could be considered. This should hypothetically lead to optimisation of anti-TNF treatment by selecting the best candidates for prolonged therapy. As a consequence, it would improve the access to anti-TNF antibodies in countries in which the assurance of long-term treatment for all patients is impossible due to pharmacoeconomic reasons.

Our analysis suggested also that mucosal healing detected after anti-TNF induction therapy could help in the prognosis of long-term remission (borderline significance); however, it was less useful when compared with the intestinal healing phenomenon. In contrast to that, the worst predictive accuracy was seen for transmural healing. Thus, it seems that endoscopy is crucial and more important than MRE for objective evaluation of $C D$ activity, although combining these two methods improves their diagnostic power, especially in the prediction of long-term results of biological treatment.

These conclusions are important, because it seems that we do not have any other better prognostic markers in CD patients treated with anti-TNF agents. In our study, only higher baseline haemoglobin concentration was significantly associated with better therapeutic outcomes. None of the other markers or parameters, assessed both before and after anti-TNF induction treatment, were statistically significantly associated with therapeutic outcomes, but there was a trend for worse response to anti-TNF agents in patients with higher inflammatory activity (higher $\mathrm{C}$-reactive protein levels, higher erythrocyte sedimentation rates). Thus, our study confirms that probably only dynamic assessment in time by using more complex diagnostic meth- 
ods (MRE, endoscopy) can provide important and useful data on the course of CD under anti-TNF therapy.

Our study has several limitations. The most important one is the low number of participants. However, this was due to very strict inclusion and exclusion criteria, which were applied in order to minimise the influence of any confounding factors for statistical analysis. Thus, our study group was very homogenous, which enabled us to perform reliable statistical analyses. On the other hand, the homogeneity of the study group (for example only patients with ileocolonic CD location) does not allow full extrapolation of the obtained data to all CD patients. Thus, the conclusions drawn in the study concern mainly patients with ileocolonic CD treated with anti-TNF antibodies.

\section{Conclusions}

To conclude, it should be emphasised that CD is a complex disease and its activity should be assessed by using "high-standard" diagnostic methods like endoscopy or MRE. This should be applied, first of all, to patients treated with biological agents, because only objective and reliable estimation of the influence of anti-TNF antibodies on the disease course could help to optimise this expensive treatment and improve its outcomes [16]. It seems that dynamic assessment of intestinal healing (mucosal healing in endoscopy and transmural healing in MRE evaluated together) is the most accurate method in predicting long-term outcomes in CD patients responding to 1-year anti-TNF therapy. Thus, the assessment of intestinal healing phenomenon could hypothetically help the clinicians to choose the best candidates for prolonged therapy. However, in order to confirm these findings, further analyses on larger patient populations are needed.

\section{Conflict of interest}

The authors declare no conflict of interest.

\section{References}

1. Cosnes J. Crohn's disease phenotype, prognosis, and long-term complications: what to expect? Acta Gastroenterol Belg 2008; 71: 303-7.

2. Siegel CA, Melmed GY. Predicting response to anti-TNF agents for the treatment of Crohn's disease. Ther Adv Gastroenterol 2009; 2: 245-51.

3. Walsh A, Palmer R, Travis S. Mucosal healing as a target of therapy for colonic inflammatory bowel disease and methods to score disease activity. Gastrointest Endosc Clin N Am 2014; 24: 367-78.

4. D'Inca R, Caccaro R. Measuring disease activity in Crohn's disease: what is currently available to the clinician. Clin Expert Gastroenterol 2014; 7: 151-61.
5. Makanyanga JC, Taylor SA. Current and future role of MR enterography in the management of Crohn's disease. AJR Am J Roentgenol 2013; 1: 56-64.

6. Tolan DJ, Greenhalgh R, Zealley IA, et al. MR enterographic manifestations of small bowel Crohn's disease. RadioGraphics 2010; 30: 367-84.

7. Łodyga M, Eder P, Bartnik W, et al. Guidelines for the management of Crohn's disease. Recommendations of the Working Group of the Polish National Consultant in Gastroenterology and the Polish Society of Gastroenterology. Prz Gastroenterol 2012; 7: 317-38.

8. Best WR, Becktel JM, Singelton JW, et al. Development of a Crohn's disease activity index. National Cooperative Crohn's Disease Study. Gastroenterology 1976; 70: 439-44.

9. Daperno M, D'Haens G, Van Assche G, et al. Development and validation of a new, simplified endoscopic activity score for Crohn's disease: the SES-CD. Gastrointest Endosc 2004; 60: 505-12.

10. Eder P, Katulska K, Lykowska-Szuber L, et al. Simple enterographic activity score for Crohn's disease: comparison with endoscopic, biochemical, and clinical findings. Pol Arch Med Wewn 2013; 7-8: 378-85.

11. Baert F, Moortgat L, Van Assche G, et al. Mucosal healing predicts sustained clinical remission in patients with early-stage Crohn's disease. Gastroenterology 2010; 138: 463-8.

12. Schnitzler F, Fidder H, Ferrante $M$, et al. Mucosal healing predicts long-term outcome of maintenance therapy with infliximab in Crohn's disease. Inflamm Bowel Dis 2009; 15: 1295-301.

13. Bjorkesten CG, Nieminen U, Sipponen T, et al. Mucosal healing at 3 months predicts long-term endoscopic remission in anti-TNF-treated luminal Crohn's disease. Scand I Gastroenterol 2013; 48: 543-51.

14. Amitai M, Ben-Horin S, Eliakim R, et al. Magnetic resonance enterography in Crohn's disease: a guide to common imaging manifestations for the IBD physician. J Crohns Colitis 2013; 8: 603-15.

15. Tielbeek JA, Lowenberg M, Bipat S, et al. Serial magnetic resonance imaging for monitoring medical therapy effects in Crohn's disease. Inflamm Bowel Dis 2013; 19: 1943-50.

16. Ben-Horin S, Kopylov U, Chowers Y. Optimizing anti-TNF treatments in inflammatory bowel disease. Autoimmun Rev 2014; 13: 24-30.

Received: 14.04.2014

Accepted: 14.06.2014 